\title{
Repurposing 57 well-known drugs for three COVID-19 targets: Mpro, Spike, RdRp
}

\author{
Bhavishya Nelakuditi*, Dr.Awantika Shrivastava** \\ *School of Computer Science and Engineering, VIT University, Vellore, India. \\ Email: bhavishya.n2017@vitstudent.ac.in \\ **Department of Molecular Modelling, Natco Research Centre, Hyderabad, India. \\ Email: nrcmm@natcopharma.co.in
}

\begin{abstract}
The pandemic that we are currently living through, caused by SARS COVID-19, changed the way we perceive deadly diseases along with our comprehension of efficacious drugs. In this paper, we compare 57 repurposable drugs from different categories like anti-inflammatory, anticancer, anticoagulant, antiviral, anti-parasitic, antidepressant, Parkinson's disease drugs, and discontinued drugs, to identify the best drugs with strongest binding affinities, that can be considered for treating COVID-19. We identified three target proteins- Main protease (PDB ID: 6LU7), Spike Glycoprotein with human receptor ACE2 (PDB ID: 6M0J), and RNA-dependent RNA polymerase (RdRp) in complex with Remdesivir (PDB ID: 7BV2) which are responsible for transcription, entrance into host's cell and viral replication respectively. We used AutoDock Vina for docking, and based our results on the binding affinity and hydrogen bond interactions. We shortlisted 15 drugs- Vilazodone, Lopinavir, Ritonavir, Darunavir, Selinexor, Etoposide, Nintedanib, Methylprednisolone, Hydrocortisone, Tolcapone, Apixaban, Rivaroxaban, Dabigatran, Betrixaban, and Amprenavir- that showcased comparable or higher affinity than Remdesivir with all of our three target proteins, and recommend these for further studies.
\end{abstract}

Keywords-Mpro, Spike, RdRp, repurposing, Remdesivir, anticoagulant, anticancer

\section{INTRODUCTION}

When the World Health Organization announced COVID-19 as a pandemic on $11^{\text {th }}$ March 2020 , there was little known about its structure or pathogenesis, except that it is similar to the severe acute respiratory syndrome coronavirus which was found in China in 2002. After a year of living through the pandemic, with five vaccines currently in use, and more underway, we remain almost equally helpless as the virus continues to mutate and spread exceedingly rapidly. The news is filled with the world undergoing destruction caused by the coronavirus. Exponential increase of people affected, 
lockdowns, shortage of vaccines, shortage of hospital beds, people suffering from breathlessness and waiting for the now almost non-existent expensive oxygen cylinders, and the blanket of grief shrouding the gray world. Working from home, wearing masks, social distancing, and constant paranoia is our new normal. Through it all, our one shining beacon of hope, Remdesivir burns brightly. But Remdesivir works by decreasing the effects of the virus only in severe cases of the COVID-19 and has no effect on mild to moderate cases. Only a fully vaccinated population can be declared healthy and safe. Africa only vaccinated $1 \%$ of their entire population. New research now predicts that coronavirus could be affected by seasons like the influenza virus, which peaks during winters [1]. Their research, which is based on the epidemiological data of the virus, hypothesized that the virus could make a regular appearance every winter and recede during the summers. Recently, Pfizer released a statement declaring the need for a third dose of the vaccine within 12 months of the second dose to reach optimum immunity. This prolongs the already steep road to normalcy and opens up new opportunities for the virus to attack even the vaccinated population.

Drug Repurposing is a cost and time effective strategy to combat the disease. While vaccines take several months to develop, and then undergo numerous testing stages, drug repurposing proves to be a safe alternative. Repurposing or Repositioning known drugs with known side-effects and dosages drastically reduces the time period of treating a disease. Commonly available drugs which can decrease the severity of the virus can help in tipping the scales of this pandemic.

Presently, there are three vital proteins that most in silico studies are based on [2]. The main protease or $3 \mathrm{CL}$ is responsible for transcription and viral replication, the spike protein plays the significant role of binding to the human ACE2 receptor which guarantees it entry to the host's cell, and RNA-dependent RNA polymerase (RdRp) or non-structural protein 12 (nsp12). The RNAdependent RNA polymerase protein or non-structural protein 12 is vital for the viral replication once it enters the host's cell. Hence, it is imperative to find suitable inhibitors to this protein which works together with other non-structural proteins such as nsp7 and nsp8 to achieve replication. Remdesivir is our reference drug since it is the only approved drug for treating COVID-19 by the FDA. 


\section{MATERIALS AND METHODS}

\subsection{TARGETS}

\subsubsection{Main Protease or Mpro}

The coronavirus 2019 main protease or Mpro became the most popular target for numerous drug repurposing studies due to its vital role in transcription and viral replication [3]. The main protease contains two identical subunits which create two active sites. Its cleavage site is located after a glutamine residue, which is exclusive to the protease and hence, does not interfere with the host cell interactions, unlike the papain-like protease or PLpro [4]. The PDB ID 6LU7 which represents the crystal structure of main protease formed using X-ray diffraction at $2.16 \mathrm{~A}$ resolution, is in complex with inhinitor $\mathrm{N} 3$ at one of the active sites. The $3 \mathrm{C}$-like proteinase cleaves the polyproteins pp1a and pp1b, giving rise to functional proteins such as nsp12, nsp7 and nsp8 which are responsible for viral replication inside the host cell. The presence of inhibitor at the binding site allows performing ligand centered docking, centered on the inhibitor N3. Remdesivir, our reference drug, formed H-bonds with residues GLY143, CYS145, and GLU166.

\subsubsection{RNA-dependent RNA polymerase}

The non-structural proteins of the coronavirus 2 are responsible for transcription and replication of the virus [5]. Nsp12 or RdRp, in complex with nsp7 and nsp8 help in improving the processivity. The PDB ID 7BV2 represents RdRp or nsp12 in complex with its other nonstructural proteins nsp7 and nsp8 with triphosphate form of of Remdesivir bound to the active site. The structure has been determined by the Electron Microscopy method at 2.50 A resolution. The structure of RdRp is similar to that of SARS-CoV, and consists of an nsp8 subunit, nsp7nsp8 heterodimer and a nsp12 core unit [6]. Since the RNA-dependent RNA polymerase has highly conserved active catalytic motifs, it makes for a popular drug target for antiviral inhibitors. The presence of Remdesivir in the active site makes it easy to determine the binding box and to execute docking studies based on the ligand. Superimposition of Remdesivir with the structure bound Remdesivir validates the chosen binding pocket. Remdesivir forms H-bonds with residues ASP760, ARG553, and ARG555.

\subsubsection{Spike Glycoprotein}

The virus that is currently causing the pandemic achieved its moniker "coronavirus" due to its crown-like spike glycoproteins. The spike protein is responsible for binding to the human 
receptor Angiotensin- converting enzyme 2, which grants it entry into the host's cell. The spike is a homotrimeric glycoprotein consisting of S1 and S2 subunits in individual monomers [7]. The PDB ID 6M0J represents the crystal structure of spike receptor binding domain bound with human receptor ACE2 using X-ray diffraction at 2.45 A resolution. Lack of an inhibitor prompts the implementation of blind docking with Remdesivir forming the H-bonds PHE40, SER44, ALA348, ASP350, ASP382 and ASN394.

\subsection{LIGANDS}

The drug candidates from ten categories: Anti-HIV drugs, CNS drugs, Antiviral drugs, Anticancer drugs, Anti-parasitic drugs, Anti-inflammatory drugs, Parkinson's disease drugs, Anti-depressants, Anticoagulants and few discontinued drugs were selected through an extensive literature survey and validated using the CORDITE (https://cordite.mathematik.uni-marburg.de/\#/) database. BIOVIA Draw was used to draw the $2 \mathrm{D}$ structure of these ligands which were converted to 3 dimensional PDB format using Frog2 software, which is inclusive of energy minimization.

Antiviral drugs are the most common repurposed drugs to treat covid-19. Remdesivir, an FDA approved drug, is the most popular repurposed drug for COVID-19 with numerous in vitro, in silico, and in vivo studies proving its efficacy against the coronavirus 2. Many countries are using it to reduce the severity of the disease. Remdesivir or GS-5734, is an antiviral drug originally meant for Hepatitis C, then repurposed for treating Ebola, and now repurposed again to treat COVID-19. Lopinavir/ Ritonavir and Favipiravir are also other widely used repurposed drugs for treating the disease [8-13]. While these drugs were used by medical practitioners in the early stages, they have now been discontinued for treating COVID-19 due to the lack of efficacy. Anti-cancer drugs are also being considered for drug repurposing strategies since the purpose of those drugs also target controlling the host environment, reducing inflammation, and inhibiting cell proliferation [14]. In using corticosteroids for treating the disease, existing in vivo research supports using Budesonide [16], Dexamethasone and Methylprednisolone [17] for further randomized clinical trials to affirm the drawn conclusions. Anti-parasitic drug, Ivermectin, which garnered a wide amount of speculation over its use in treating the disease, has proven to be effective in preventing the replication of the virus in in vitro, but requires further in vivo studies to ascertain its efficacy [18]. Chloroquine and Hydroxychloroquine, on the other hand, have been in use to treat patients during the early stages of the pandemic, before they were prohibited due to low efficacy and strong sideeffects [19]. Anticoagulants are required to reduce the complications caused by the disease like 
thrombosis, but no research has been published yet which shows the efficiency of anticoagulants in inhibiting the viral proteins [20]. There are several clinical trials currently underway and few which already concluded that the antidepressants Fluvoxamine and Fluoxetine aid in reducing the inflammation caused by the disease and limit the deterioration of patients affected [21], though official approval is required for these drugs to be considered as a potential treatment option for the disease. The figure 1 below lists the flowchart of methodology.

\subsection{HARDWARE AND SOFTWARE SPECIFICATIONS}

The research has been conducted with the following system specifications. Processor: Intel ® Core тM i5-6400 CPU @ 2.70 GHz, Installed RAM: 4.00 GB, System type: 64-bit operating system, x64based processor. The Windows specifications are Edition: Windows 10 pro, Version: 1909.

BIOVIA draw was implemented for drawing the 2D structures of fifty-seven ligands. Frog2 software converts the drawn 2D structures of these ligands to 3D with built-in energy minimization. Prediction of binding pockets of targets is done using PrankWeb and CASTp software. AutoDock Vina is required for the docking process, with PMV tools 1.6 showing the graphical representation. BIOVIA Discovery Studio is used to visualize the docked conformation of the ligand in the binding site as well as to analyze features like H-bonds. 


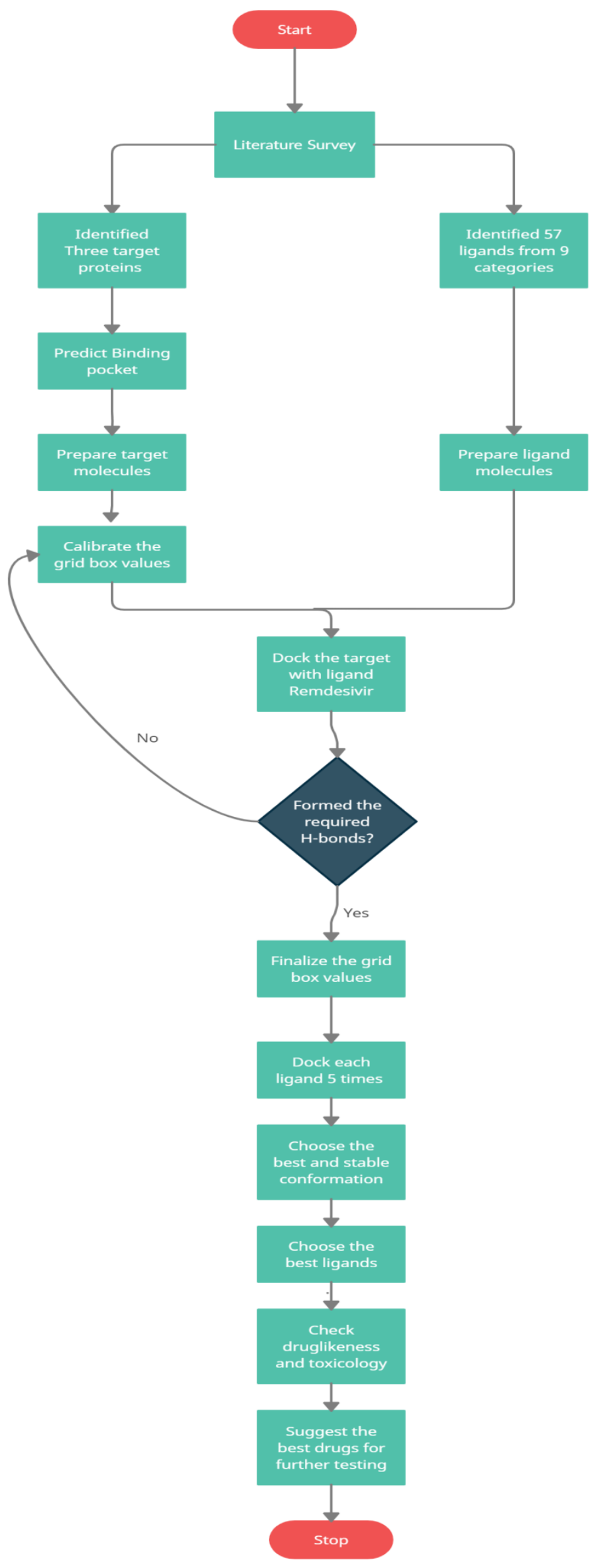

Figure 1: Flowchart of methodology 


\subsection{DOCKING USING AUTODOCK VINA}

\subsubsection{Preparation of target molecules}

We downloaded the PDB structure of the target proteins 6LU7, 6M0J, and 7BV2 from Protein Data Bank. Minimized the energy of the proteins to achieve stable conformations. Deleted all water molecules except the ones in the binding pocket. According to recent studies, polarized water molecules in binding pockets aid in increasing the binding affinity of the ligand rather than reducing it, as they act as part of the protein and influence the ligand binding conformation [22]. We then added all hydrogens with bond order and Kollman charges. Since crystal files generally lack hydrogen atoms, adding them is necessary to optimize the system. Save the files in PDBQT format.

\subsubsection{Preparation of Ligand Molecule}

Convert the drawn 2D structure of ligand in SMILES or Mol format to PDB using Frog2 software with in-built energy minimization. Frog2 also adds the hydrogens inclusively, so just remove water ions present, if any. Next, add stereo chemical information, if it lacks stereoisomers, followed by adding Gasteiger charges. Go to the ligand option of ADT tools, and choose the ligand. Adjust the rotatable bonds as necessary ( $<10$ is preferable), and save the ligand in PDBQT format.

\subsubsection{Choosing Grid parameters}

The receptor is to be rigid and the ligand, flexible. Select the macromolecule and adjust the grid box values appropriately to calculate the surface area. This helps in analyzing the binding pockets of the receptor for docking. Since RdRp (7BV2) and Mpro (6LU7) have crystal structures with a ligand and inhibitor complex respectively, we set the binding box centered on them and superimposed our docked output with the respective ligand to choose the optimum grid box values. The grid box values of 7BV2 are $x$-center $=100.84, y$-center=92.664, z-center=105.585, and x-points $=24, y$ points $=12$, and z-points $=10$, the spacing set to $1 \mathrm{~A}$. The center grid box values of 6LU7 are $\mathrm{x}$ center $=-10.749, \mathrm{y}$-center $=12.46, \mathrm{z}$-center $=68.91$, and the sizes are $\mathrm{x}$-points $=24, \mathrm{y}$-points $=24$, and $\mathrm{z}$ points=22, the spacing set to $1 \mathrm{~A}$. Due to the lack of crystal structures of spike glycoprotein with ligands, we performed blind docking for the target protein 6M0J. Blind docking is to choose the grid values in such a way as to cover the entirety of the protein. The grid box values chosen for $6 \mathrm{M} 0 \mathrm{~J}$ are $\mathrm{x}$-center $=-23.088, \mathrm{y}$-center $=18.676, \mathrm{z}$-center $=-27.106$, and $\mathrm{x}$-points $=64, \mathrm{y}$-points $=66$, and $\mathrm{z}$ points $=72$, the spacing set to $1 \mathrm{~A}[10]$. 


\subsubsection{Evaluation of Results}

Dock each ligand multiple times to reach a stable conformation. Select the stable conformation with lowest binding affinity and analyze the H-bonds.

\section{RESULTS}

\subsection{RNA-dependent RNA polymerase}

The target molecule 7BV2, which is the protein RNA-dependent RNA polymerase ( $R d R p)$, in complex with Remdesivir, has the binding pocket with residues ARG555, TRP617, ASP618, TYR619, LYS621, CYS622, ASP623, THR680, SER682, ASP684, THR687, ALA688, ASN691, SER759, ASP760, ASP761, LYS798, THRP800, and GLU811. Since the crystal is in complex with the ligand Remdesivir, after deleting the heteroatom, ligand-based docking was performed and the resulting conformations were superimposed with the molecule's Remdesivir to confirm the binding pocket. Remdesivir bound to RdRp in the binding pocket with a binding affinity of $-7.4 \mathrm{kcal} / \mathrm{mol}$ and made the following H-bonds: ASP760, ARG553, ARG555, and SER682, which is taken as the reference. 7BV2 exhibited highest binding affinity to Etoposide, an antineoplastic drug used to treat leukemia, ovarian cancer, lung cancer, testicular cancer and lymphoma. It exhibited a binding affinity of $-8.4 \mathrm{kcal} / \mathrm{mol}$ with 7BV2 and made H-bonds with residues ARG555 and ASN691. Figure 2 shows the docked result of Etoposide with 7BV2 visualized using BIOVIA Discovery Studio. Dabigatran exhibited the binding affinity $-8.2 \mathrm{kcal} / \mathrm{mol}$, follows Etoposide. 7BV2 showed the least binding affinity with Amantadine at $-4.6 \mathrm{kcal} / \mathrm{mol}$. It also showed low binding affinities with Pramiprexole, Rasagiline, and Cyamemazine- as proved by its lack of H-bonds. 7BV2 bound well to anticoagulants and anti-cancer drugs in general, while Parkinson's disease drugs displayed low affinity towards 7BV2.

\subsection{Spike Glycoprotein}

6M0J, which is the spike glycoprotein in complex with ACE2, has the binding pocket PHE40, THR347, ALA348, ASP350, GLU375, HIS378, ASP382, TYR385, ASN394, and HIS 401. Spike protein docked with Remdesivir has a binding affinity of $-7.5 \mathrm{kcal} / \mathrm{mol}$ and made H-bonds with the residues PHE40, SER44, ALA348, ASP350, ASP382, and ASN394. 6M0J exhibited highest binding affinity to Elbasvir (anti-viral) at $-11.6 \mathrm{kcal} / \mathrm{mol}$, and made $\mathrm{H}$-bonds with the residues TRP69, 
ASP350, HIS374, and SER409. Figure 3 shows the docked result of Elbasvir with 6M0J visualized using BIOVIA Discovery Studio.

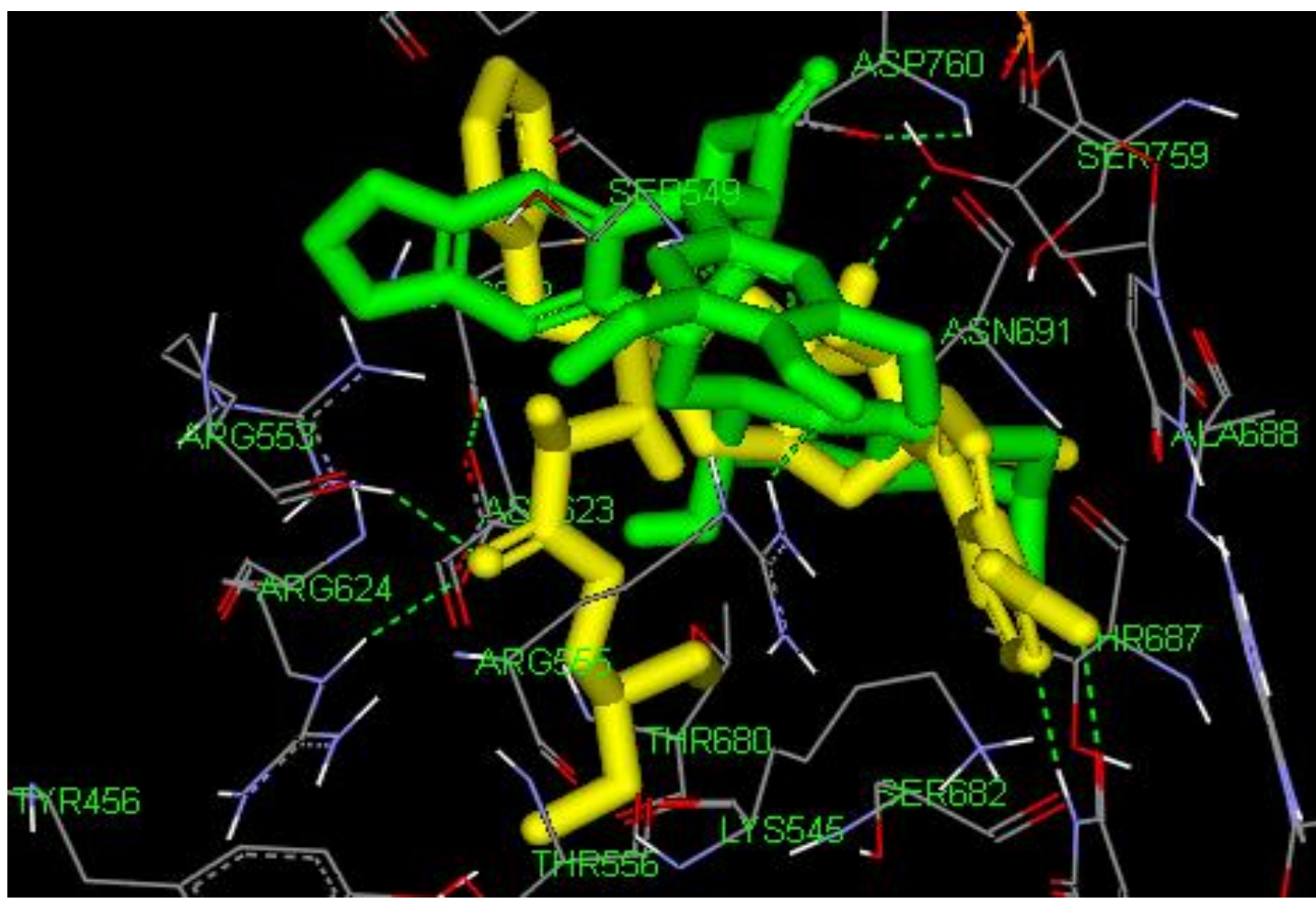

Figure 2: Docked result of Etoposide (Green) with 7BV2 (RdRp), superimposed with Remdesivir (yellow). H-bonds are visualized as dotted green lines using Discovery Studio.

Vilazodone, Bromocriptine (Parkinson's disease drug), Lopinavir (antiviral), and Selinexor (anticancer drug) expressed the binding affinities -10.0, -9.5, -9.2, and $-9.2 \mathrm{kcal} / \mathrm{mol}$ respectively. 6M0J showed the least binding affinity with Amantadine at $-4.9 \mathrm{kcal} / \mathrm{mol}$, as proven by the lack of H-bonds. It also showed low binding affinities with Levamisole and Favipiravir- which wasn't docked at the required binding pocket. Discontinued drugs Telaprevir and Amprenavir also showed promising results with binding affinities -8.5 and $-8.1 \mathrm{kcal} / \mathrm{mol}$ respectively. $6 \mathrm{M} 0 \mathrm{~J}$ bound well to anticoagulants, and anti-cancer drugs in general while Parkisnon's disease drugs displayed low affinity towards $6 \mathrm{M} 0 \mathrm{~J}$.

\subsection{Main Protease}

Main protease or Mpro represented by the PDB ID 6LU7 has the binding pocket THR25, THR26, LEU27, HIS41, MET49, PHE140, LEU141, ASN142, GLY143, SER144, CYS145, HIS163, HIS164, MET165, GLU166, ASP187, ARG188, and GLN189. When docked with Remdesivir, Hbonds were created with the residues THR24, THR25, THR26, CYS44, THR45, SER46, ASN142, 
GLY143, SER144, CYS145, and HIS164. Mpro has the highest binding affinity with Vilazodone at $-8.8 \mathrm{kcal} / \mathrm{mol}$ and made H-bonds with the residues SER46, THR25, and THR45. Figure 4 shows the docked result of Vilazodone with 6LU7 visualized using BIOVIA Discovery Studio. Selinexor, Etoposide and Betrixaban, exhibited higher affinities than Remdesivir at -8.4, -8.4,and -8.3 kcal/mol respectively. Hydrocortisone, Bromocriptine, Methylprednisolone, Lopinavir, Darunavir, Nintedanib, Rivaroxaban, and Amprenavir showed comparable affinity with Remdesivir at -8.1, 8.1, -8.0, -7.9, -7.9, -7.8, -7.8, -7.7, and $-7.7 \mathrm{kcal} / \mathrm{mol}$ respectively. Amantadine showed the least binding affinity with 6LU7 at $-4.8 \mathrm{kcal} / \mathrm{mol}$. Anticoagulants bound well to 6LU7 while Parkinson's disease drugs showed least binding affinities towards the main protease. Table 1 showcases the binding affinities of the 57 drugs with respective target proteins. The hydrogen bonds made by each ligand with the protein are displayed in Table 2. Docking results of best ligands for each protein are included in supplementary files.

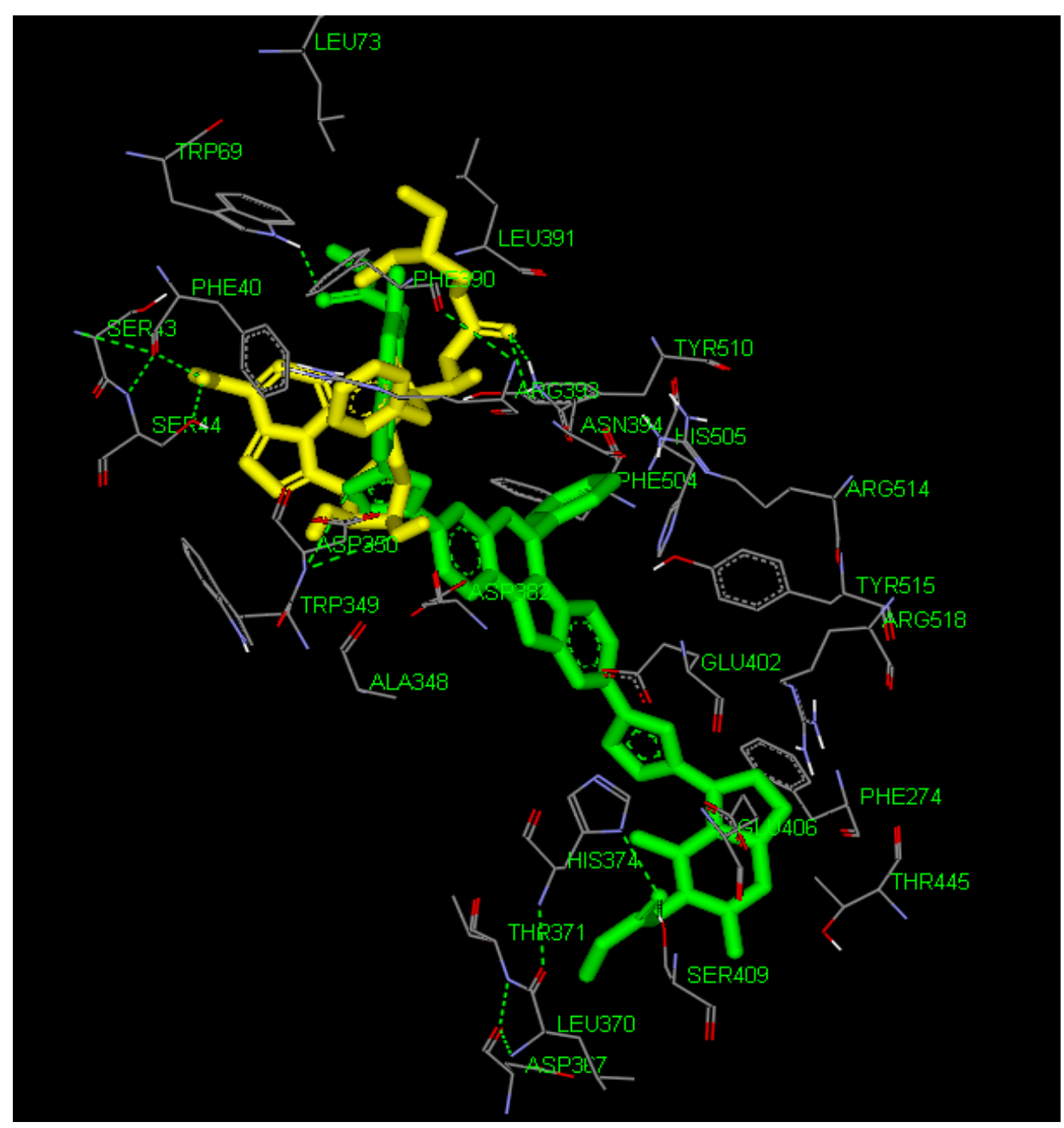

Figure 3: Docked result of Elbasvir (Green) with 6M0J (Spike), superimposed with Remdesivir (yellow). H-bonds are visualized as dotted green lines using Discovery Studio. 


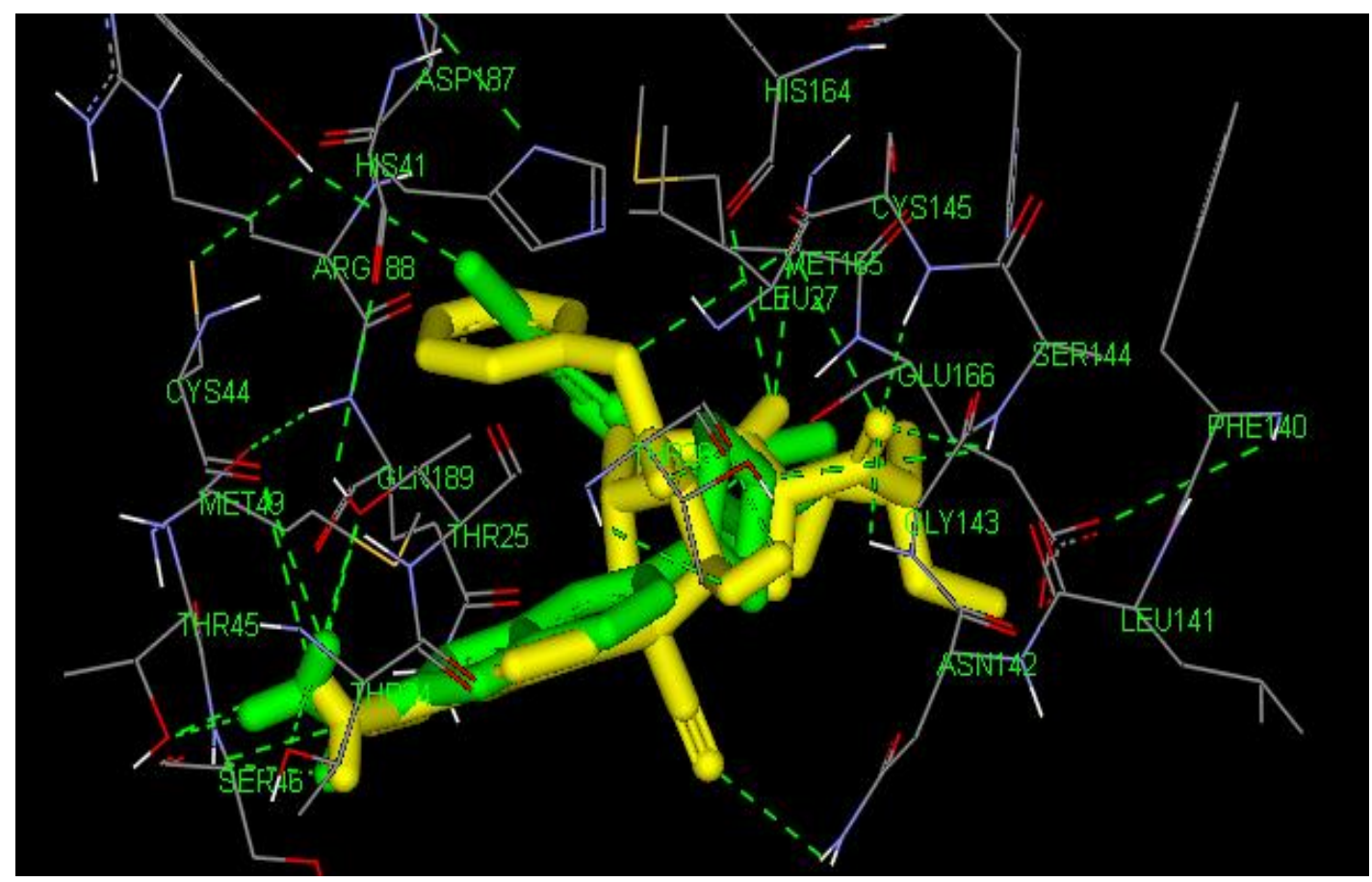

Figure 4: Docked result of Vilazodone (Green) with 6LU7 (Mpro), superimposed with Remdesivir (yellow). H-bonds are visualized as dotted green lines using Discovery Studio.

\begin{tabular}{|l|l|l|l|}
\hline \multicolumn{1}{|c|}{ Drug } & \multicolumn{1}{|c|}{$\begin{array}{c}\text { BA of 7BV2 } \\
\mathbf{k c a l} / \mathbf{m o l}\end{array}$} & \multicolumn{1}{c|}{$\begin{array}{c}\text { BA of 6M0J } \\
\mathbf{k c a l} / \mathbf{m o l}\end{array}$} & \multicolumn{1}{c|}{$\begin{array}{c}\text { BA of 6LU7 } \\
\mathbf{k c a l} / \mathbf{m o l}\end{array}$} \\
\hline $\begin{array}{l}\text { Reference- } \\
\text { Remdesivir }\end{array}$ & -7.4 & -7.5 & -8.1 \\
\hline Anti-HIV agents & & -8.7 & -7.5 \\
\hline Etravirine & -6.5 & -8.3 & -7.1 \\
\hline Dapivirine & -7.1 & -8.8 & -7.4 \\
\hline Rilpivirine & -6.8 & -6.3 & -6.2 \\
\hline Lersivirine & -5.8 & & \\
\hline CNS Drugs & & -6.9 & -6.3 \\
\hline Escitalopram & -5.6 & -10.0 & -8.8 \\
\hline Vilazodone & -7.6 & -8.0 & -7.5 \\
\hline Periciazine & -6.1 & -6.4 & -6.5 \\
\hline Cyamemazine & -5.5 & -7.4 & -7.1 \\
\hline Zaleplon & -6.8 & -8.1 & -7.3 \\
\hline Donitriptan & -7.5 & & -7.9 \\
\hline Antiviral drugs & & -9.2 & \\
\hline Lopinavir & -7.6 & & \\
\hline
\end{tabular}




\begin{tabular}{|c|c|c|c|}
\hline Ritonavir & -7.1 & -8.4 & -7.5 \\
\hline Favipiravir & -5.7 & -5.9 & -5.1 \\
\hline Ribavirin & -6.8 & -6.8 & -6.4 \\
\hline Darunavir & -7.9 & -7.9 & $\begin{array}{l}-7.9 \\
\end{array}$ \\
\hline Arbidol & -6.2 & -7.3 & -6.5 \\
\hline Elbasvir & -5.7 & -11.6 & -7.5 \\
\hline Sofosbuvir & -7.8 & -7.2 & -7.2 \\
\hline Galidesivir & -6.6 & -6.7 & -6.9 \\
\hline \multicolumn{4}{|l|}{ Anti-cancer Drugs } \\
\hline Thalidomide & -7.1 & $\begin{array}{ll}-6.8 \\
\end{array}$ & -6.7 \\
\hline Selinexor & -8.0 & -9.2 & -8.4 \\
\hline Etoposide & -8.4 & -7.9 & -8.4 \\
\hline Nintedanib & -7.4 & -8.5 & -7.8 \\
\hline \multicolumn{4}{|l|}{$\begin{array}{l}\text { Anti-parasitic } \\
\text { Drugs }\end{array}$} \\
\hline Ivermectin & -5.7 & -8.8 & -7.3 \\
\hline Chloroquine & -5.8 & -6.1 & -5.8 \\
\hline Levamisole & -5.7 & -5.9 & -5.6 \\
\hline Nitazoxanide & -6.7 & -6.7 & -6.3 \\
\hline Hydroxychloroquine & -5.8 & -6.1 & -5.8 \\
\hline \multicolumn{4}{|l|}{$\begin{array}{l}\text { Anti-inflammatory } \\
\text { drugs }\end{array}$} \\
\hline Budesonide & -6.0 & -8.5 & -7.1 \\
\hline Dexamethasone & -6.6 & $\begin{array}{ll}-7.8 \\
\end{array}$ & -7.3 \\
\hline Methylprednisolone & -7.1 & -7.9 & -8.0 \\
\hline Prednisone & -6.9 & -7.8 & -7.1 \\
\hline Hydrocortisone & -6.9 & -7.4 & -8.1 \\
\hline Prednisolone & -6.9 & -7.5 & -7.3 \\
\hline \multicolumn{4}{|l|}{$\begin{array}{l}\text { Parkinson's } \\
\text { Disease Drugs }\end{array}$} \\
\hline Amantadine & -4.6 & -4.9 & -4.8 \\
\hline Levodopa & -5.8 & -6.3 & -5.7 \\
\hline Entacapone & -5.9 & -6.2 & -6.3 \\
\hline
\end{tabular}




\begin{tabular}{|c|c|c|c|}
\hline Tolcapone & -7.2 & -7.1 & -7.6 \\
\hline Bromocriptine & -5.7 & -9.5 & -8.1 \\
\hline Ropinirole & -5.6 & -6.3 & -5.6 \\
\hline Pramiprexole & -4.8 & -5.3 & -5.5 \\
\hline Rotigotine & -5.3 & -6.0 & -6.1 \\
\hline Selegiline & -5.2 & -5.7 & -5.1 \\
\hline Rasagiline & -4.9 & -6.1 & -5.4 \\
\hline \multicolumn{4}{|c|}{ Anti-Depressants } \\
\hline Fluvoxamine & -6.3 & -6.1 & -5.9 \\
\hline Fluoxetine & -6.7 & -7.0 & -6.6 \\
\hline \multicolumn{4}{|c|}{ Anti-Coagulants } \\
\hline Apixaban & -8.0 & -7.8 & -7.5 \\
\hline Edoxaban & \begin{tabular}{|l|}
-7.7 \\
\end{tabular} & -8.5 & -7.3 \\
\hline Rivaroxaban & \begin{tabular}{|l|}
-7.7 \\
\end{tabular} & -7.9 & -7.8 \\
\hline Dabigatran & -8.2 & -8.6 & -7.5 \\
\hline Betrixaban & \begin{tabular}{|l|}
-8.0 \\
\end{tabular} & -7.9 & -8.3 \\
\hline \multicolumn{4}{|c|}{ Discontinued Drugs } \\
\hline Vidarabine & \begin{tabular}{|l|}
-7.2 \\
\end{tabular} & -6.7 & -6.3 \\
\hline Zalcitabine & -5.9 & -6.2 & -5.6 \\
\hline Amprenavir & \begin{tabular}{|l|}
-7.2 \\
\end{tabular} & -8.1 & -7.7 \\
\hline Boceprevir & -6.6 & -7.6 & -6.8 \\
\hline Telaprevir & -5.7 & -8.5 & -7.0 \\
\hline
\end{tabular}

Table 1: Binding affinities of 57 ligands with 7BV2, 6M0J, and 6LU7

\begin{tabular}{|l|l|l|l|}
\hline Drug & H-bonds 7BV2 & H-Bonds 6M0J & H-bonds 6LU7 \\
\hline Reference-Remdesivir & ASP760 & PHE40 & THR24 \\
& ARG553 & SER44 & THR25 \\
& ARG555 & ALA348 & THR26 \\
& SER682 & ASP350 & CYS44 \\
& & ASP382 & THR45 \\
& & ASN394 & SER46 \\
& & & ASN142 \\
& & & GLY143 \\
& & & SER144 \\
\hline
\end{tabular}




\begin{tabular}{|c|c|c|c|}
\hline & & & $\begin{array}{l}\text { CYS145 } \\
\text { HIS164 }\end{array}$ \\
\hline \multicolumn{4}{|l|}{ Anti-HIV agents } \\
\hline Etravirine & $\begin{array}{l}\text { ASP760 } \\
\text { ASP623 }\end{array}$ & $\begin{array}{l}\text { ASP350 } \\
\text { ASP382 } \\
\text { HIS401 } \\
\text { ASN394 }\end{array}$ & $\begin{array}{l}\text { GLY143 } \\
\text { GLU166 } \\
\text { HIS164 } \\
\text { THR190 }\end{array}$ \\
\hline Dapivirine & ASP760 & $\begin{array}{l}\text { ASP382 } \\
\text { ASP350 } \\
\text { ARG393 } \\
\text { GLU402 }\end{array}$ & $\begin{array}{l}\text { GLU166 } \\
\text { CYS145 }\end{array}$ \\
\hline Rilpivirine & ASP760 & $\begin{array}{l}\text { ASP350 } \\
\text { ARG393 }\end{array}$ & $\begin{array}{l}\text { GLU166 } \\
\text { HIS164 } \\
\text { ASP187 }\end{array}$ \\
\hline Lersivirine & $\begin{array}{l}\text { ARG555 } \\
\text { CYS622 }\end{array}$ & $\begin{array}{l}\text { ASP350 } \\
\text { ARG393 } \\
\text { ASN394 }\end{array}$ & $\begin{array}{l}\text { SER144 } \\
\text { GLU166 }\end{array}$ \\
\hline \multicolumn{4}{|l|}{ CNS Drugs } \\
\hline Escitalopram & $\begin{array}{l}\text { ARG555 } \\
\text { ASN691 }\end{array}$ & $\begin{array}{l}\text { SER47 } \\
\text { ARG393 } \\
\text { ASN394 }\end{array}$ & $\begin{array}{l}\text { THR26 } \\
\text { PHE140 } \\
\text { GLU166 }\end{array}$ \\
\hline Vilazodone & $\begin{array}{l}\text { SER682 } \\
\text { ASP760 }\end{array}$ & $\begin{array}{l}\text { ASP350 } \\
\text { GLN102 }\end{array}$ & $\begin{array}{l}\text { SER46 } \\
\text { THR25 } \\
\text { THR45 }\end{array}$ \\
\hline Periciazine & ARG555 & $\begin{array}{l}\text { VAL343 } \\
\text { HIS345 }\end{array}$ & $\begin{array}{l}\text { THR190 } \\
\text { PHE140 } \\
\text { HIS163 } \\
\text { GLU166 }\end{array}$ \\
\hline Cyamemazine & No H-bonds & $\begin{array}{l}\text { VAL343 } \\
\text { HIS345 }\end{array}$ & NO H-BONDS \\
\hline Zaleplon & $\begin{array}{l}\text { ASN691 } \\
\text { ARG553 } \\
\text { SER759 }\end{array}$ & ASN394 & $\begin{array}{l}\text { GLY143 } \\
\text { THR26 }\end{array}$ \\
\hline Donitriptan & ASN691 & LYS74 & GLY143 \\
\hline
\end{tabular}




\begin{tabular}{|c|c|c|c|}
\hline & $\begin{array}{l}\text { SER759 } \\
\text { LYS621 }\end{array}$ & $\begin{array}{l}\text { ASP350 } \\
\text { ASN103 }\end{array}$ & THR25 \\
\hline \multicolumn{4}{|l|}{ Antiviral drugs } \\
\hline Lopinavir & $\begin{array}{l}\text { ARG553 } \\
\text { ARG555 }\end{array}$ & $\begin{array}{l}\text { ASN394 } \\
\text { GLU375 } \\
\text { ALA348 }\end{array}$ & $\begin{array}{l}\text { ASN142 } \\
\text { GLU166 } \\
\text { MET165 } \\
\text { GLN189 } \\
\text { ASN142 } \\
\text { ARG188 }\end{array}$ \\
\hline Ritonavir & $\begin{array}{l}\text { ARG553 } \\
\text { ARG555 }\end{array}$ & $\begin{array}{l}\text { ASN394 } \\
\text { TYR385 } \\
\text { ASP382 }\end{array}$ & $\begin{array}{l}\text { SER144 } \\
\text { CYS145 } \\
\text { GLU166 } \\
\text { GLN189 } \\
\text { HIS163 } \\
\text { THR26 }\end{array}$ \\
\hline Favipiravir & $\begin{array}{l}\text { ARG555 } \\
\text { SER759 } \\
\text { ASP760 }\end{array}$ & $\begin{array}{l}\text { LYS353 } \\
\text { GLY496 } \\
\text { HIS34 }\end{array}$ & $\begin{array}{l}\text { SER144 } \\
\text { CYS145 }\end{array}$ \\
\hline Ribavirin & ARG555 & $\begin{array}{l}\text { TRP556 } \\
\text { ASN210 } \\
\text { ALA396 }\end{array}$ & $\begin{array}{l}\text { GLU166 } \\
\text { HIS164 } \\
\text { HIS163 }\end{array}$ \\
\hline Darunavir & $\begin{array}{l}\text { ARG555 } \\
\text { ASP623 } \\
\text { ASN691 }\end{array}$ & $\begin{array}{l}\text { ASP350 } \\
\text { ASN394 }\end{array}$ & $\begin{array}{l}\text { GLU166 } \\
\text { THR25 } \\
\text { GLY143 } \\
\text { CYS44 } \\
\text { THR24 }\end{array}$ \\
\hline Arbidol & ARG555 & ALA348 & NO H-BONDS \\
\hline Elbasvir & $\begin{array}{l}\text { ARG555 } \\
\text { LYS621 }\end{array}$ & $\begin{array}{l}\text { TRP69 } \\
\text { ASP350 } \\
\text { HIS374 } \\
\text { SER409 }\end{array}$ & $\begin{array}{l}\text { THR26 } \\
\text { ALA191 } \\
\text { THR26 } \\
\text { ASN119 }\end{array}$ \\
\hline Sofosbuvir & $\begin{array}{l}\text { ARG553 } \\
\text { ASN691 } \\
\text { ARG555 }\end{array}$ & $\begin{array}{l}\text { ASP350 } \\
\text { ARG514 } \\
\text { ASP382 }\end{array}$ & $\begin{array}{l}\text { THR26 } \\
\text { GLN189 }\end{array}$ \\
\hline
\end{tabular}




\begin{tabular}{|c|c|c|c|}
\hline Galidesivir & ASP760 & $\begin{array}{l}\text { ASP382 } \\
\text { TYR385 } \\
\text { ASN394 }\end{array}$ & $\begin{array}{l}\text { SER144 } \\
\text { LEU141 }\end{array}$ \\
\hline \multicolumn{4}{|l|}{ Anti-cancer Drugs } \\
\hline Thalidomide & $\begin{array}{l}\text { ARG555 } \\
\text { ASN691 } \\
\text { ASP760 }\end{array}$ & ASN394 & $\begin{array}{l}\text { GLY143 } \\
\text { GLU166 } \\
\text { LEU141 }\end{array}$ \\
\hline Selinexor & ARG553 & $\begin{array}{l}\text { ARG393 } \\
\text { ASP350 }\end{array}$ & $\begin{array}{l}\text { GLY143 } \\
\text { THR190 } \\
\text { GLN192 } \\
\text { GLU166 } \\
\text { SER144 } \\
\text { HIS163 } \\
\text { ASN142 }\end{array}$ \\
\hline Etoposide & $\begin{array}{l}\text { ARG555 } \\
\text { ASN691 }\end{array}$ & ALA348 & GLY143 \\
\hline Nintedanib & $\begin{array}{l}\text { ARG555 } \\
\text { ASP623 }\end{array}$ & $\begin{array}{l}\text { SER47 } \\
\text { ALA348 } \\
\text { ASP382 } \\
\text { ASN394 }\end{array}$ & $\begin{array}{l}\text { GLY143 } \\
\text { HIS41 }\end{array}$ \\
\hline \multicolumn{4}{|l|}{ Anti-parasitic Drugs } \\
\hline Ivermectin & $\begin{array}{l}\text { LYS551 } \\
\text { ARG555 } \\
\text { LYS621 } \\
\text { CYS622 }\end{array}$ & $\begin{array}{l}\text { ASP350 } \\
\text { ASN394 }\end{array}$ & $\begin{array}{l}\text { GLY143 } \\
\text { THR24 }\end{array}$ \\
\hline Chloroquine & ASN691 & $\begin{array}{l}\text { ASP350 } \\
\text { HIS401 }\end{array}$ & $\begin{array}{l}\text { GLY143 } \\
\text { HIS164 }\end{array}$ \\
\hline Levamisole & ARG555 & ASP350 & NO H-BONDS \\
\hline Nitazoxanide & $\begin{array}{l}\text { ARG553 } \\
\text { ARG555 } \\
\text { ASN691 } \\
\text { SER759 }\end{array}$ & $\begin{array}{l}\text { ASP350 } \\
\text { ASP382 }\end{array}$ & $\begin{array}{l}\text { SER144 } \\
\text { CYS145 }\end{array}$ \\
\hline Hydroxychloroquine & ARG553 & ASN394 & ASP187 \\
\hline Anti-inflammatory drugs & & & \\
\hline
\end{tabular}




\begin{tabular}{|c|c|c|c|}
\hline Budesonide & ARG553 & $\begin{array}{l}\text { ASP350 } \\
\text { ASP382 }\end{array}$ & $\begin{array}{l}\text { THR26 } \\
\text { HIS41 }\end{array}$ \\
\hline Dexamethasone & $\begin{array}{l}\text { ARG553 } \\
\text { THR680 }\end{array}$ & $\begin{array}{l}\text { ASP350 } \\
\text { ASP382 }\end{array}$ & $\begin{array}{l}\text { GLU166 } \\
\text { LEU141 }\end{array}$ \\
\hline Methylprednisolone & $\begin{array}{l}\text { LYS621 } \\
\text { ASN691 } \\
\text { SER759 }\end{array}$ & $\begin{array}{l}\text { ASP350 } \\
\text { ALA348 }\end{array}$ & $\begin{array}{l}\text { GLY143 } \\
\text { SER144 } \\
\text { HIS163 }\end{array}$ \\
\hline Prednisone & ARG555 & $\begin{array}{l}\text { ASP350 } \\
\text { ALA348 }\end{array}$ & $\begin{array}{l}\text { ASN142 } \\
\text { LEU141 }\end{array}$ \\
\hline Hydrocortisone & $\begin{array}{l}\text { ARG555 } \\
\text { ASN691 } \\
\text { SER759 } \\
\text { ASP760 }\end{array}$ & $\begin{array}{l}\text { ASP350 } \\
\text { ASP382 } \\
\text { HIS401 }\end{array}$ & $\begin{array}{l}\text { GLY143 } \\
\text { LEU141 } \\
\text { SER144 }\end{array}$ \\
\hline Prednisolone & $\begin{array}{l}\text { ARG555 } \\
\text { THR680 }\end{array}$ & $\begin{array}{l}\text { ASP382 } \\
\text { ASP350 }\end{array}$ & \\
\hline Parkinson's Disease & & & \\
\hline Amantadine & ASN691 & No H-bonds & NO H-BONDS \\
\hline Levodopa & $\begin{array}{l}\text { ARG555 } \\
\text { SER682 } \\
\text { ASP760 }\end{array}$ & $\begin{array}{l}\text { ASN394 } \\
\text { ASP350 }\end{array}$ & $\begin{array}{l}\text { LEU141 } \\
\text { HIS164 }\end{array}$ \\
\hline Entacapone & $\begin{array}{l}\text { ARG555 } \\
\text { THR680 } \\
\text { ASN691 }\end{array}$ & $\begin{array}{l}\text { ALA348 } \\
\text { ASP382 }\end{array}$ & $\begin{array}{l}\text { SER144 } \\
\text { LEU141 }\end{array}$ \\
\hline Tolcapone & $\begin{array}{l}\text { ARG553 } \\
\text { ARG555 } \\
\text { ARG624 } \\
\text { THR680 } \\
\text { THR556 }\end{array}$ & $\begin{array}{l}\text { ASP382 } \\
\text { ASP350 } \\
\text { ALA348 }\end{array}$ & $\begin{array}{l}\text { SER144 } \\
\text { CYS145 } \\
\text { LEU141 }\end{array}$ \\
\hline Bromocriptine & LYS621 & $\begin{array}{l}\text { ASN394 } \\
\text { ASP350 }\end{array}$ & $\begin{array}{l}\text { GLU166 } \\
\text { ASN142 } \\
\text { SER144 }\end{array}$ \\
\hline Ropinirole & $\begin{array}{l}\text { ARG553 } \\
\text { THR556 } \\
\text { ASP452 }\end{array}$ & $\begin{array}{l}\text { ASP350 } \\
\text { TYR385 }\end{array}$ & $\begin{array}{l}\text { MET165 } \\
\text { ARG188 }\end{array}$ \\
\hline
\end{tabular}




\begin{tabular}{|c|c|c|c|}
\hline Pramiprexole & $\begin{array}{l}\text { SER759 } \\
\text { ASP760 }\end{array}$ & $\begin{array}{l}\text { ASP350 } \\
\text { ASP382 } \\
\text { ALA348 }\end{array}$ & $\begin{array}{l}\text { GLY143 } \\
\text { HIS41 }\end{array}$ \\
\hline Rotigotine & $\begin{array}{l}\text { ARG555 } \\
\text { ARG553 }\end{array}$ & SER43 & LEU141 \\
\hline Selegiline & No H-bonds & No H-bonds & NO H-BONDS \\
\hline Rasagiline & $\begin{array}{l}\text { SER759 } \\
\text { ASP760 }\end{array}$ & $\begin{array}{l}\text { PHE390 } \\
\text { ARG393 }\end{array}$ & HIS164 \\
\hline \multicolumn{4}{|c|}{ Anti-Depressants } \\
\hline Fluvoxamine & $\begin{array}{l}\text { ASP760 } \\
\text { SER682 } \\
\text { ARG624 } \\
\text { TYR456 }\end{array}$ & $\begin{array}{l}\text { TYR385 } \\
\text { ASN394 } \\
\text { ASP382 }\end{array}$ & $\begin{array}{l}\text { HIS41 } \\
\text { GLY143 } \\
\text { LEU141 }\end{array}$ \\
\hline Fluoxetine & $\begin{array}{l}\text { THR556 } \\
\text { ALA558 } \\
\text { ARG624 } \\
\text { ASP623 }\end{array}$ & $\begin{array}{l}\text { ASP382 } \\
\text { TYR385 }\end{array}$ & SER144 \\
\hline \multicolumn{4}{|c|}{ Anti-Coagulants } \\
\hline Apixaban & $\begin{array}{l}\text { ARG553 } \\
\text { ARG555 }\end{array}$ & $\begin{array}{l}\text { SER47 } \\
\text { ASP350 }\end{array}$ & $\begin{array}{l}\text { GLU166 } \\
\text { CYS145 }\end{array}$ \\
\hline Edoxaban & $\begin{array}{l}\text { ARG555 } \\
\text { ASP623 }\end{array}$ & ASP350 & $\begin{array}{l}\text { GLY143 } \\
\text { GLU166 } \\
\text { GLN189 }\end{array}$ \\
\hline Rivaroxaban & $\begin{array}{l}\text { ARG555 } \\
\text { CYS622 } \\
\text { ASP623 } \\
\text { SER759 }\end{array}$ & $\begin{array}{l}\text { LYS74 } \\
\text { ASN394 }\end{array}$ & $\begin{array}{l}\text { THR26 } \\
\text { CYS145 } \\
\text { SER144 } \\
\text { GLU166 } \\
\text { HIS164 }\end{array}$ \\
\hline Dabigatran & $\begin{array}{l}\text { ARG553 } \\
\text { THR556 } \\
\text { ASP623 }\end{array}$ & $\begin{array}{l}\text { ASN394 } \\
\text { GLY395 } \\
\text { ASP206 } \\
\text { ALA348 }\end{array}$ & $\begin{array}{l}\text { ARG188 } \\
\text { GLY143 } \\
\text { GLU166 }\end{array}$ \\
\hline Betrixaban & $\begin{array}{l}\text { ARG553 } \\
\text { ARG555 }\end{array}$ & $\begin{array}{l}\text { ASP350 } \\
\text { PHE40 }\end{array}$ & $\begin{array}{l}\text { GLY143 } \\
\text { CYS145 }\end{array}$ \\
\hline
\end{tabular}




\begin{tabular}{|c|c|c|c|}
\hline & ASP623 & SER44 & HIS164 \\
\hline \multicolumn{4}{|l|}{ Discontinued Drugs } \\
\hline Vidarabine & $\begin{array}{l}\text { ARG555 } \\
\text { ASN691 } \\
\text { SER759 } \\
\text { SER682 }\end{array}$ & $\begin{array}{l}\text { ALA348 } \\
\text { TYR385 }\end{array}$ & $\begin{array}{l}\text { CYS145 } \\
\text { LEU141 }\end{array}$ \\
\hline Zalcitabine & $\begin{array}{l}\text { ARG555 } \\
\text { ASP760 }\end{array}$ & $\begin{array}{l}\text { ASN394 } \\
\text { ASP350 }\end{array}$ & $\begin{array}{l}\text { SER144 } \\
\text { CYS145 } \\
\text { GLU166 }\end{array}$ \\
\hline Amprenavir & $\begin{array}{l}\text { ARG555 } \\
\text { ASN691 }\end{array}$ & $\begin{array}{l}\text { ASP350 } \\
\text { TYR385 } \\
\text { SER43 }\end{array}$ & $\begin{array}{l}\text { GLY143 } \\
\text { CYS145 } \\
\text { HIS164 } \\
\text { THR24 } \\
\text { THR25 }\end{array}$ \\
\hline Boceprevir & $\begin{array}{l}\text { ARG555 } \\
\text { ASN691 } \\
\text { ASP623 } \\
\text { SER759 }\end{array}$ & $\begin{array}{l}\text { ASN394 } \\
\text { ALA348 }\end{array}$ & THR26 \\
\hline Telaprevir & $\begin{array}{l}\text { ARG555 } \\
\text { LYS621 } \\
\text { ASN691 }\end{array}$ & $\begin{array}{l}\text { ASN394 } \\
\text { ALA348 } \\
\text { ASP350 }\end{array}$ & $\begin{array}{l}\text { GLY143 } \\
\text { GLU166 } \\
\text { HIS163 }\end{array}$ \\
\hline
\end{tabular}

Table 2: H-bonds of 57 ligands with 7BV2, 6M0J, and 6LU7

\section{DISCUSSION}

We repurposed commonly available drugs among various categories such as antiviral, anticancer, anticoagulants, anti-inflammatory, Parkinson's disease drugs, antidepressants and discontinued drugs to aid in the fight against COVID-19. We chose three target proteins: Main Protease(PDB ID:6LU7), Spike Glycoprotein (PDB ID: 6M0J), and RNA-dependent RNA polymerase (PDB ID:7BV2) to facilitate our work. We also chose Remdesivir as our reference drug since it is a popular and widely used drug to treat the disease and docked it against our three targets RdRp, Spike and Mpro initially with binding affinities $-7.4 \mathrm{kcal} / \mathrm{mol},-7.5 \mathrm{kcal} / \mathrm{mol}$ and $-8.1 \mathrm{kcal} / \mathrm{mol}$ respectively. Through our research, we identified 15 drugs in total which either showed comparable or greater affinity to all of our chosen target proteins as compared with Remdesivir. For the target 7BV2, Etoposide exhibited highest binding affinity at $-8.4 \mathrm{kcal} / \mathrm{mol}$ and formed the H-bonds 
ARG555 and ASN691. The target 6M0J bound well to Elbasvir at $-11.6 \mathrm{kcal} / \mathrm{mol}$ and made the hydrogen bonds with residues TRP69, ASP350, HIS374, and SER409 while Mpro displayed the highest affinity with Vilazodone at $-8.8 \mathrm{kcal} / \mathrm{mol}$ with H-bonds SER46, THR25 and THR45. While Elbasvir and Bromocriptine showed comparable affinities with 6M0J and 6LU7, they did not dock well with 7BV2. Vilazodone showed higher affinity than Remdesivir with all three targets- RdRp, Spike, and Mpro at $-7.6 \mathrm{kcal} / \mathrm{mol},-10.0 \mathrm{kcal} / \mathrm{mol}$, and $-8.8 \mathrm{kcal} / \mathrm{mol}$ respectively. Antiviral drugs Lopinavir (7BV2:6M0J:6LU7::-7.6 kcal/mol, $-9.2 \mathrm{kcal} / \mathrm{mol},-7.9 \mathrm{kcal} / \mathrm{mol}$ ), Ritonavir (-7.1 $\mathrm{kcal} / \mathrm{mol},-8.4 \mathrm{kcal} / \mathrm{mol},-7.5 \mathrm{kcal} / \mathrm{mol})$, and Darunavir $(-7.9 \mathrm{kcal} / \mathrm{mol},-7.9 \mathrm{kcal} / \mathrm{mol},-7.9 \mathrm{kcal} / \mathrm{mol})$ showed comparable affinity with our reference drug while anti-parasitic and Parkinson's disease drugs showed least affinity with all three of our target proteins. Anticancer drugs Selinexor (-8.0 $\mathrm{kcal} / \mathrm{mol},-9.2 \mathrm{kcal} / \mathrm{mol},-8.4 \mathrm{kcal} / \mathrm{mol})$, Etoposide $(-8.4 \mathrm{kcal} / \mathrm{mol},-7.9 \mathrm{kcal} / \mathrm{mol},-8.4 \mathrm{kcal} / \mathrm{mol})$, Nintedanib (-7.4 kcal/mol, -8.5 kcal/mol, -7.8 kcal/mol), and anticoagulant drugs , Rivaroxaban ($7.7 \mathrm{kcal} / \mathrm{mol},-7.9 \mathrm{kcal} / \mathrm{mol},-7.8 \mathrm{kcal} / \mathrm{mol})$, Dabigatran $(-8.2 \mathrm{kcal} / \mathrm{mol},-8.6 \mathrm{kcal} / \mathrm{mol},-7.5 \mathrm{kcal} / \mathrm{mol})$, and Betrixaban $(-8.0 \mathrm{kcal} / \mathrm{mol},-7.9 \mathrm{kcal} / \mathrm{mol},-8.3 \mathrm{kcal} / \mathrm{mol})$ also showed promising results with the three target proteins. Two anti-inflammatory drugs Methylprednisolone $(-7.1 \mathrm{kcal} / \mathrm{mol},-7.9$ $\mathrm{kcal} / \mathrm{mol},-8.0 \mathrm{kcal} / \mathrm{mol}$ ) and Hydrocortisone (-6.9 kcal $/ \mathrm{mol},-7.4 \mathrm{kcal} / \mathrm{mol},-8.1 \mathrm{kcal} / \mathrm{mol})$, one Parkinson's disease drug Tolcapone $(-7.2 \mathrm{kcal} / \mathrm{mol},-7.1 \mathrm{kcal} / \mathrm{mol},-7.6 \mathrm{kcal} / \mathrm{mol})$, one discontinued drug Amprenavir (-7.2 kcal/mol, $-8.1 \mathrm{kcal} / \mathrm{mol},-7.7 \mathrm{kcal} / \mathrm{mol})$ also proved comparable with Remdesivir. The ligands bound well to $6 \mathrm{M} 0 \mathrm{~J}-$ spike glycoprotein. In general, anticancer and anticoagulant drugs bound well to all of the three target proteins.

\section{ADME PREDICTION}

The prediction of various physiochemical and pharmacokinetic properties was done using SwissADME (http://www.swissadme.ch/index.php). Ritonavir and Etoposide do not satisfy the Lipinski rule of 5 due to Molecular weight and NorO violations, which exceeded the acceptable range. Vilazodone, Methylprednisolone, Hydrocortisone, Tolcapone, Apixaban, Rivaroxaban, Dabigatran and Betrixaban satisfied all of Lipinski's rules and are deemed orally active. Lopinavir, Darunavir, Selinexor, Nintedanib, and Amprenavir also satisfy Lipinski's rules with one violation of high molecular weight, making them orally active. Table 3 shows the Lipnski's rules for the shortlisted drugs. 


\begin{tabular}{|l|l|l|l|l|l|}
\hline Drug & $\begin{array}{l}\text { Mass } \\
(<\mathbf{5 0 0} \\
\text { Dalton }) \\
\text { g/mol }\end{array}$ & $\begin{array}{l}\text { Hydrogen } \\
\text { Bond } \\
\text { Acceptors(< } \\
\mathbf{1 0})\end{array}$ & $\begin{array}{l}\text { Hydrogen } \\
\text { Bond } \\
\text { Donors(<5 } \\
\text { (1) }\end{array}$ & $\begin{array}{l}\text { MLOGP( } \\
<\mathbf{5})\end{array}$ & $\begin{array}{l}\text { rupinski } \\
\text { violations }\end{array}$ \\
\hline Vilazodone & 441.52 & 4 & 2 & 1.6 & Yes-0 \\
\hline Lopinavir & 632.83 & 5 & 4 & 2.93 & Yes-1 \\
\hline Ritonavir & 724.98 & 7 & 4 & 1.8 & No-2 \\
\hline Darunavir & 549.68 & 8 & 3 & 1.18 & Yes-1 \\
\hline Selinexor & 443.31 & 11 & 2 & 1.18 & Yes-1 \\
\hline Etoposide & 588.56 & 13 & 3 & -0.14 & No-2 \\
\hline Nintedanib & 541.64 & 7 & 2 & 2.36 & Yes-1 \\
\hline Methylprednisolone & 375.48 & 5 & 3 & 1.52 & Yes-0 \\
\hline Hydrocortisone & 363.47 & 5 & 3 & 1.39 & Yes-0 \\
\hline Tolcapone & 274.25 & 5 & 3 & 0.97 & Yes-0 \\
\hline Apixaban & 459.50 & 5 & 1 & 2.03 & Yes-0 \\
\hline Rivaroxaban & 435.88 & 5 & 3 & 1.41 & Yes-0 \\
\hline Dabigatran & 476.55 & 6 & 1.63 & Yes-0 \\
\hline Betrixaban & 453.92 & 5 & 2.56 & Yes-0 \\
\hline Amprenavir & 509.66 & 7 & 1.14 & Yes-1 \\
\hline
\end{tabular}

Table 3: Lipinski rules for shortlisted drugs from SwissADME

\section{Cytochrome inhibitors}

The cytochromes belonging to the P450 subfamily are enzymes responsible for a host of metabolic activities. One key cytochrome is the CYP3A4 which is responsible for oxidation of small molecules leading to their excretion from the body. Lopinavir, Ritonavir, Darunavir, Selinexor, and Amprenavir inhibit this cytochrome but do not inhibit the rest from the same subfamily suggesting low gastrointestinal absorption [23]. Table 4 showcases the cytochrome inhibitors for chosen drugs.

\begin{tabular}{|l|l|l|l|l|l|}
\hline Drug & $\begin{array}{l}\text { CYP1A2 } \\
\text { inhibitor }\end{array}$ & $\begin{array}{l}\text { CYP2C1 } \\
\mathbf{9} \\
\text { inhibitor }\end{array}$ & $\begin{array}{l}\text { CYP2C9 } \\
\text { inhibitor }\end{array}$ & $\begin{array}{l}\text { CYP2D6 } \\
\text { inhibitor }\end{array}$ & $\begin{array}{l}\text { CYP3A4 } \\
\text { inhibitor }\end{array}$ \\
\hline Vilazodone & no & yes & yes & yes & yes \\
\hline Lopinavir & no & no & no & no & yes \\
\hline
\end{tabular}




\begin{tabular}{|l|l|l|l|l|l|}
\hline Ritonavir & no & no & no & no & yes \\
\hline Darunavir & no & no & no & no & yes \\
\hline Selinexor & no & no & no & no & yes \\
\hline Etoposide & no & no & no & yes & no \\
\hline Nintedanib & no & yes & yes & yes & no \\
\hline Methylprednisolone & no & no & no & no & no \\
\hline Hydrocortisone & no & no & no & no & no \\
\hline Tolcapone & no & no & no & no & no \\
\hline Apixaban & no & yes & yes & yes & yes \\
\hline Rivaroxaban & no & yes & yes & no & yes \\
\hline Dabigatran & no & yes & no & no & no \\
\hline Betrixaban & no & yes & no & no & no \\
\hline Amprenavir & no & no & no & no & yes \\
\hline
\end{tabular}

Table 4: Cytochrome Inhibitors for shortlisted drugs from SwissADME

\section{Bioavailability}

Oral bioavailability of a drug depends on six properties as predicted by SwissADME. The lipophilicity of a drug as determined by XLOGP3 should be in the range -0.7 and +5.0 , the size as determined by the mass should be in between 150 and $500 \mathrm{~g} / \mathrm{mol}$, the polarity as determined by TPSA should exist between 20 and $130 \AA^{2}$, the solubility as determined by Log S (ESOL) should not be greater than 6 , saturation as determined by fraction csp3 must be greater than 0.25 , and finally, the flexibility as determined by the number of rotatable bonds must not exceed 9.

Taking these properties into consideration, the drugs available orally are Vilazodone. Methylprednisolone, Hydrocortisone, Apixaban, and Rivaroxaban. Out of these, Vilazodone are moderately soluble while Methylprednisolone, Hydrocortisone and Rivaroxaban are soluble. Table 5 showcases the bioavailability factors of the shortlisted drugs.

\begin{tabular}{|c|c|c|c|c|c|c|c|}
\hline Drug & $\begin{array}{l}\text { Lipophilicit } \\
\mathbf{y} \\
\text { XLOGP3 }\end{array}$ & $\begin{array}{l}\text { Mass } \\
(<500 \\
\text { Dalton }) \\
\text { g/mol }\end{array}$ & TPSA & $\begin{array}{l}\text { Log S } \\
\text { (ESO } \\
\text { L) }\end{array}$ & $\begin{array}{l}\text { ESOL } \\
\text { Class }\end{array}$ & $\begin{array}{l}\text { Fraction } \\
\text { csp3 }\end{array}$ & $\begin{array}{l}\text { Rotatable } \\
\text { bonds }\end{array}$ \\
\hline Vilazodone & 3.98 & 444.55 & 102.29 & -5.05 & $\begin{array}{l}\text { Moderately } \\
\text { soluble }\end{array}$ & 0.31 & 7 \\
\hline
\end{tabular}




\begin{tabular}{|c|c|c|c|c|c|c|c|}
\hline Lopinavir & 5.92 & 632.83 & 120 & -6.66 & $\begin{array}{l}\text { Poorly } \\
\text { soluble }\end{array}$ & 0.43 & 17 \\
\hline Ritonavir & 6.05 & 724.98 & 202.26 & -7.02 & $\begin{array}{l}\text { Poorly } \\
\text { soluble }\end{array}$ & 0.43 & 22 \\
\hline Darunavir & 2.94 & 549.68 & 148.8 & -4.48 & $\begin{array}{l}\text { Moderately } \\
\text { soluble }\end{array}$ & 0.52 & 13 \\
\hline Selinexor & 2.94 & 445.32 & 97.62 & -4.48 & $\begin{array}{l}\text { Moderately } \\
\text { soluble }\end{array}$ & 0.12 & 8 \\
\hline Etoposide & 0.6 & 588.56 & 160.83 & -3.75 & Soluble & 0.55 & 5 \\
\hline Nintedanib & 4.3 & 541.64 & 101.47 & -5.7 & $\begin{array}{l}\text { Moderately } \\
\text { soluble }\end{array}$ & 0.26 & 9 \\
\hline $\begin{array}{l}\text { Methylpredni } \\
\text { solone }\end{array}$ & 1.95 & 375.48 & 94.83 & -3.26 & Soluble & 0.73 & 2 \\
\hline $\begin{array}{l}\text { Hydrocotison } \\
\mathrm{e}\end{array}$ & 1.61 & 363.47 & 94.83 & -2.98 & Soluble & 0.81 & 2 \\
\hline Tolcapone & 2.83 & 274.25 & 107.19 & -3.57 & Soluble & 0.07 & 3 \\
\hline Apixaban & 2.24 & 459.5 & 110.76 & -4.14 & $\begin{array}{l}\text { Moderately } \\
\text { soluble }\end{array}$ & 0.28 & 5 \\
\hline Rivaroxaban & 2.49 & 435.88 & 116.42 & -4 & Soluble & 0.32 & 6 \\
\hline Dabigatran & 1.7 & 476.55 & 150.22 & -3.65 & Soluble & 0.16 & 10 \\
\hline Betrixaban & 3.56 & 453.92 & 107.41 & -4.72 & $\begin{array}{l}\text { Moderately } \\
\text { soluble }\end{array}$ & 0.13 & 9 \\
\hline Amprenavir & 2.89 & 509.66 & 139.57 & -4.22 & $\begin{array}{l}\text { Moderately } \\
\text { soluble }\end{array}$ & 0.48 & 13 \\
\hline
\end{tabular}

Table 5: Bioavailability factors for shortlisted drugs from SwissADME

\section{CONCLUSION}

Vaccines are a preventative form of medicine. While effective in decreasing the effect caused due to the exposure of the disease, it cannot help people who are already suffering. At this stage, we are going through the second wave of the COVID-19 pandemic with three FDA-approved vaccines for emergency use- Pfizer-BioNTech vaccine, Moderna Vaccine, Janssen vaccine- and one approved repurposed drug- Remdesivir. While most of the world is taking part in vaccination drives and various lockdowns, India and Brazil are currently the most affected countries ravaged by this 
disease, with thousands dying every day and a severe lack of vaccines, equipment, drugs, and hospital facilities to deal with the pandemic. Repurposing commonly available and commonly used drugs provides a shortcut to understanding and decreasing the effects of the disease. Since repurposing involves already approved drugs, it cuts short the time required to formulate, assessing the side-effects and undergoing various clinical trials. Since Remdesivir is the only approved drug to treat the disease, its scarcity and price pose a massive hindrance to overcoming this pandemic. Out of the 57 repurposed drugs, we shortlisted fifteen based on the binding affinity and H-bond interactions, which are likely to prove beneficial in fighting the disease. These drugs are: Vilazodone (CNS drug), Lopinavir (Antiviral), Ritonavir (Antiviral), Darunavir (Antiviral), Selinexor (Anticancer), Etoposide (Anticancer), Nintedanib (Anticancer), Methylprednisolone (antiinflammatory), Hydrocortisone (anti-inflammatory), Tolcapone (Parkinson's disease drug), Apixaban (anticoagulant), Rivaroxaban (anticoagulant), Dabigatran (anticoagulant), Betrixaban (anticoagulant), and Amprenavir (discontinued drug). We recommend these drugs for further in vitro and in vivo studies to determine their efficacy in fighting the disease.

\section{ACKNOWLEDGMENTS}

We would like to thank Dr.K.Amala, General manager- R \& D, Natco Research Centre, for giving us the opportunity to work on this paper and guiding us throughout the course of the internship. I am also thankful to my university, Vellore Institute of Technology, Vellore, India, for equipping me with the knowledge and resources that led me to this endeavor.

\section{REFERENCES}

[1] A. Audi, M. AlIbrahim, M. Kaddoura, G. Hijazi, H. M. Yassine, and H. Zaraket, "Seasonality of Respiratory Viral Infections: Will COVID-19 Follow Suit?,” Front. Public Heal., vol. 8, no. September, pp. 1-8, 2020, doi: 10.3389/fpubh.2020.567184.

[2] S. Dotolo, A. Marabotti, A. Facchiano, and R. Tagliaferri, "A review on drug repurposing applicable to COVID-19," Brief. Bioinform., vol. 22, no. 2, pp. 726-741, 2021, doi: 10.1093/bib/bbaa288.

[3] Z. Jin et al., "Structure of Mpro from SARS-CoV-2 and discovery of its inhibitors," Nature, vol. 582, no. 7811, pp. 289-293, 2020, doi: 10.1038/s41586-020-2223-y.

[4] S. Ullrich and C. Nitsche, "The SARS-CoV-2 main protease as drug target," Bioorganic Med. Chem. Lett., vol. 30, no. 17, p. 127377, 2020, doi: 10.1016/j.bmcl.2020.127377.

[5] W. Yin et al., "Structural basis for inhibition of the SARS-CoV-2 RNA polymerase by suramin," Nat. Struct. Mol. Biol., vol. 28, no. 3, pp. 319-325, 2021, doi: 10.1038/s41594-021-00570-0.

[6] D. Design, "Repurposing Anti-Cancer Drugs for COVID-19 Treatment," pp. 5045-5058, 2020. 
[7] F. F. Fleming, L. Yao, P. C. Ravikumar, L. Funk, and B. C. Shook, “412) 3966031 Fax. (412) 3965683 † Mylan Pharmaceuticals Inc., 781 Chestnut Ridge Rd,” vol. 53, no. 22, pp. 7902-7917, 1947, doi: 10.1021/jm100762r.Nitrile-Containing.

[8] Y. Jiang, W. Yin, and H. E. Xu, "RNA-dependent RNA polymerase: Structure, mechanism, and drug discovery for COVID-19," Biochem. Biophys. Res. Commun., vol. 538, no. xxxx, pp. 47-53, 2021, doi: 10.1016/j.bbrc.2020.08.116.

[9] J. Lan et al., "Structure of the SARS-CoV-2 spike receptor-binding domain bound to the ACE2 receptor," Nature, vol. 581, no. 7807, pp. 215-220, 2020, doi: 10.1038/s41586-020-2180-5.

[10] V. R. Naik et al., "Remdesivir (GS-5734) as a therapeutic option of 2019-nCOV main protease-in silico approach," J. Biomol. Struct. Dyn., vol. 0, no. 0, pp. 1-14, 2020, doi: 10.1080/07391102.2020.1781694.

[11] N. Jena, "Identification of Potent Drugs and Antiviral Agents for the Treatment of the SARSCoV-2 Infection," ChemRxiv, no. 1, 2020, doi: 10.26434/chemrxiv.12330599.

[12] K. G. Kalamatianos, "Drug Repurposing for Coronavirus (COVID-19): In Silico Screening of Known Drugs Against the SARS-CoV-2 Spike Protein Bound to Angiotensin Converting Enzyme 2 (ACE2) (6M0J)," Anal. Chem. Toxicol. Lab., I.Y.A, vol. 2, 2020, doi: 10.26434/chemrxiv.12857678.v2.

[13] R. V. Chikhale et al., "Sars-cov-2 host entry and replication inhibitors from Indian ginseng: an insilico approach,” J. Biomol. Struct. Dyn., vol. 0, no. 0, pp. 1-12, 2020, doi: 10.1080/07391102.2020.1778539.

[14] M. T. Rehman, M. F. AlAjmi, and A. Hussain, "Natural Compounds as Inhibitors of SARS-CoV2 Main Protease (3CLpro): A Molecular Docking and Simulation Approach to Combat COVID19," Curr. Pharm. Des., vol. 26, 2020, doi: 10.2174/1381612826999201116195851.

[15] S. Farabi, N. R. Saha, M. Hasanuzzaman, N. A. Khan, and M. S. Haque, "Prediction of sars-cov-2 main protease inhibitors in medicinal plant derived compounds by molecular docking approach," J. Adv. Biotechnol. Exp. Ther., vol. 3, no. Special Issue 4, pp. 76-96, 2020, doi: 10.5455/JABET.2020.D159.

[16] S. Ramakrishnan et al., "Inhaled budesonide in the treatment of early COVID-19 (STOIC): a phase 2, open-label, randomised controlled trial," Lancet Respir. Med., 2021, doi: https://doi.org/10.1101/2021.02.04.21251134.

[17] M. H. Ahmed and A. Hassan, "Dexamethasone for the Treatment of Coronavirus Disease (COVID-19): a Review,” SN Compr. Clin. Med., vol. 2, no. 12, pp. 2637-2646, 2020, doi: 10.1007/s42399-020-00610-8.

[18] L. Caly, J. D. Druce, M. G. Catton, D. A. Jans, and K. M. Wagsta, "Since January 2020 Elsevier has created a COVID-19 resource centre with free information in English and Mandarin on the novel coronavirus COVID- 19 . The COVID-19 resource centre is hosted on Elsevier Connect , the company' s public news and information," Antiviral Res., vol. 178, no. January, p. 104787, 2020.

[19] M. S. Khuroo, "Since January 2020 Elsevier has created a COVID-19 resource centre with free information in English and Mandarin on the novel coronavirus COVID- 19 . The COVID-19 resource centre is hosted on Elsevier Connect, the company' s public news and information," Int. J. Antimicrob. Agents, vol. 56, no. January, p. 13, 2020.

[20] J. S. Rico-Mesa, D. Rosas, A. Ahmadian-Tehrani, A. White, A. S. Anderson, and R. Chilton, "The Role of Anticoagulation in COVID-19-Induced Hypercoagulability," Curr. Cardiol. Rep., vol. 22, no. 7, pp. 1-6, 2020, doi: 10.1007/s11886-020-01328-8.

[21] E. J. Lenze et al., "Fluvoxamine vs Placebo and Clinical Deterioration in Outpatients with Symptomatic COVID-19: A Randomized Clinical Trial," JAMA - J. Am. Med. Assoc., vol. 324, no. 22, pp. 2292-2300, 2020, doi: 10.1001/jama.2020.22760.

[22] M. Maurer and C. Oostenbrink, "Water in protein hydration and ligand recognition," J. Mol. Recognit., vol. 32, no. 12, pp. 1-19, 2019, doi: 10.1002/jmr.2810.

[23] M. D. Lima de Oliveira and K. M. Teixeira de Oliveira, "Comparative Computational Study of 
SARS-CoV-2 Receptors Antagonists from Already Approved Drugs," 2020, doi: 10.26434/chemrxiv.12044538.v1. 